\title{
Lexical Translation in Movies: A Comparative Analysis of Persian Dubs and Subtitles Through CDA
}

\author{
Noie Saber ${ }^{1,}$, , Jafar Pour Fariba ${ }^{2}$ \\ ${ }^{1}$ Faculty of Translation and English Literature, South Tehran Branch of Islamic Azad University, Tehran, Iran \\ ${ }^{2}$ Faculty of Persian Literature, North Tehran Branch of Islamic Azad University, Tehran, Iran
}

Email address:

saber.noie@yahoo.com (N. Saber), jafarpourfariba@yahoo.com (J. P. Fariba)

${ }^{*}$ Corresponding author

\section{To cite this article:}

NoieSaber, Jafar Pour Fariba. Lexical Translation in Movies: A Comparative Analysis of Persian Dubs and Subtitles Through CDA. Arabic Language, Literature \& Culture. Vol. 3, No. 3, 2018, pp. 29-36. doi: 10.11648/j.allc.20180303.12

Received: August 11, 2018; Accepted: August 27, 2018; Published: October 11, 2018

\begin{abstract}
There are two major types of film translation: dubbing and subtitling; each of them interferes with the original were discussed text to a different extent. On one hand, dubbing known to be the method that modifies the source text largely and thus makes it familiar to the target audience through domestication. The impact of translated movies has already been emphasized by quiet a number of researchers. The present study aimed to investigate the strategies of the Iranian subtitlers and dubbers of English movies in rendering English words. To this aim, three theoretical frameworks were employed: the strategies proposed by Venuti, and Newmark's classification used first by researcher and then, researcher went back to the Van Dijk's Critical Discourse Analysis (CDA), in the part dominance. The aim was to find which strategies were the most prevalently used by Iranian subtitlers and dubbers and to see which model fitted these attempts the best. A movie was investigated: the Girl with the Dragon Tattoo. Through a qualitative content analysis, distribution of strategies was found and reported in frequencies and percentages. They were crossed - compared between the three frameworks. The result showed that which strategies of each model were used more. The results of this study may pave the way for future research in literary translation and help translation instructors and translation trainees as well in translation classes.
\end{abstract}

Keywords: Critical Discourse Analysis (CDA), Domestication, Dub, Foreignization, Subtitle

\section{Introduction}

In the age of globalization, digitalization, and dominance of media, audiovisual translation (AVT) can play an increasingly important role in communication across cultures and languages. Movie is a mass medium and translated movies are the only kind of translations, which are received by a mass audience. People have become much more interested in movies over many years. Despite being an important part of the audiovisual market, translation of movies still lacks scholarly attention in Iran.

Many of the studies done on subtitling and dubbing focused on translation strategies used in these processes and the frequency and adequacy of each strategy. These studies tried to classify different translation strategies and find linguistic dimensions affecting the quality of subtitling and dubbing. They also tried to suggest possible solutions to enhance the quality of subtitling and dubbing. These studies have rarely worked on translating of lexical items directly.

Translation oflexical items is one of the most difficult areas facing a translator particularly while translating movies. If translators want their target language text to be accepted and understood by their audience, they should behave in accordance with what is expected and meaningful in the target culture. The problem of replacing or translating words can be interpreted not only as a purely linguistic matter but also as psychological, literary, cognitive and so on [1].

There are two major types of film translation: dubbing and subtitling. On one hand, dubbing known to be the method that modifies the source text largely and thus makes it familiar to the target audience through domestication. It is the methods in which the foreign dialogue is adjusted to the mouth and movements of the actor in the film and its aim is seen as making the audience feel as if they were listening to actors actually speaking the target language. On the other hand, subtitling i.e. supplying a translation of the spoken source language dialogue into the target language in the form 
of synchronized captions, usually at the bottom of the screen, is the form that alters the source text to the least possible extent and enables the target audience to be aware of its 'foreignness' at all times [2].

Replacing words with more familiar ones shows a lack of respect toward other cultures. This also deprives readers of the chance to realize the wealth of cultural diversity that surrounds them. Translators should be aware of realm of readers adapting words to the target culture. Audience's lack of background knowledge gets often problematic. As a result, close attention must be paid to movies, which are to be translated for people. Accordingly, translators usually use different strategies to deal with lexical items and these strategies can engender different communicative effects on the respective audience [3].

The gap is further extended to the realm of movies. Considering the above mentioned issues, the present research decided to fill the gap and address the following question:

(1) Which strategies and what distribution were used in translating lexical items by Iranian dubbers and subtitlers of English movies based on Venuti's strategies of domestication and foreignization?

(2) Which strategies and what distribution were used in translating lexical items by Iranian dubbers and subtitlers of English movies Newmark's classification of culture-specific items?

(3) According to CDA defined by Van Dijk (in the part of dominance), during translating and dubbing of the lexical items in movies, which strategy is dominance?

The following section discusses some of the related literature on translation of movies. Regarding strategies used in translation of inter-lingual subtitling, Ghaemi and Benyamin (2011)tried to identify the inter-lingual subtitling strategies employed to translate English subtitles into Persian and determine their frequency. The researchers selected an English-Persian parallel corpus of 932 frames of five English feature films [4]. The selected films were among different genres. To analyze the data, the researchers used the classification of inter-lingual subtitling strategies proposed by Gottlieb (1992) as the theoretical framework. The analysis of the result showed that different strategies were used in transferring the expressions of the original films. The findings of the study showed that all Gottlieb's (1992) proposed strategies were used in English-Persian subtitling of English feature films with some degree of variation. They concluded that the film genre played a crucial role in the variation of the used strategy. They also concluded that the subtitles transferred as much information as possible by translating the dialogues completely and accurately [5].

Vahid Dastjerdi (2010) tried to classify different types of expansions used in subtitling and investigate the appropriateness and inappropriateness of the application of each type considering the time and space constraints which are peculiar to subtitling. In line with the goal of the study, the researchers selected three English films. The rationale behind their selection was that the quality of the Persian subtitles was excellent. The researchers concluded that subtitles are just one channel of information in a polysemiotic text like a film which includes other semiotic channels such as pictures, music, and sound effect. Thus, subtitlers should not include everything in the subtitles, and should let viewers receive part of the information from other channels [6].

Subtitling is often assumed to be a solely linguistic operation which involves the translation of dialogue into written captions. However, from a viewer's perspective subtitled productions include more than just linguistic information. In addition to subtitles, viewers have to process film images in order to establish a coherent narrative. Both types of information must be received through the same visual channel. In this regard, De Linde \& Kay (2016) examined a number of significant linguistic and nonlinguistic features of subtitles and film which potentially affect the way viewers watch subtitled productions. Nonlinguistic features were examined in the context of recent studies on eye-movement behavior, including a comparative study involving deaf and hearing viewers conducted by the authors [7].

In a study conducted by Pelsmaekers (2002) the researchers set out to examine empirically how humorous irony fares in the constrained form of translation that subtitling was, and whether these observations could be related to known humor translation and subtitling strategy [8]. Drawing on a speech act approach to irony the researchers studied 211 humorous ironic utterances and their Dutch subtitles from twelve episodes of the 'Blackadder' series. While a quantitative approach showed that nearly all these subtitles retained some degree of ironic potential, it was also found that in about two thirds of these cases verbal ironic cues were influenced by the translation in interesting ways. A qualitative analysis showed that on the whole, the Dutch subtitles were often more explicitly critical. This means that from the known strategies of irony translation only a few turned out to be very important in these data. Part of this could be explained by subtitling strategies reported in the literature, which generally seemed to work against the preservation of a particular kind of ironic cues, i.e. those that were found to have an interpersonal rather than referential function.

As for subtitling, it is defined as the translation of the spoken (or written) source text of an audiovisual product, usually at the bottom of the screen [9]. Gambier (1994) defined subtitling as transferring language from longer units to shorter one, from spoken language to written text, from one language to another. He added an interpretation of verbal speech combined with numerous other cultural and socio symbolic signs or with other types of semiotic system [10].

Fernandez (2006) outlined a translation oriented parametrical proposal for contrastive analysis taking account of micro and macro linguistic factors [11]. Bertills (2003) also claimed that the main dilemma of translating proper names in literature derives from their complicated nature, as they are culture-specific items. A good translation needs to take into account both the linguistic and textual aspects of the 
name as well as their role in the narrative and in the target language [12].

The authenticity of fictional dialogue is widely held to play a pivotal role in shaping the audience's perception of the quality of a film. Yet the factors that account for the authenticity of both original and dubbed film conversations remain largely under-researched. In this regard, PerezGonzalez'es (2007) paper began by outlining key contributions from the fields of stylistics, film studies and corpus-based translation studies that have enhanced our understanding of the specific nature and dynamics of fictional dialogue and its translation [13]. A common assumption that underpins these approaches is that the success of the narrative and characterization-enhancing resources deployed in a film is contingent on the build-up of interpersonal alignments through a combination of prefabricated orality and spontaneous-sounding conversation. And yet both film theory and dubbing studies have so far focused on phenomena that take place within a single turn-attalk and hence neglected the study of the sequential dimension of film dialogue.

\section{Method}

The movie "The girl with the dragon tattoo" along with its corresponding Persian versions in both dubbed and subtitled versions was chosen as the materials of the study. It was produced in 2011and subtitled, and dubbed into Persian in the same year. The researcher worked on the whole dialogue of the movie. The movie prepared in the DVD format, because the scripts prepared from the internet did not match with the original dialogues and sequences in many parts. There were some criteria in choosing this movie; one of the reasons for such selection was that both versions of its Persian translation, i.e., the dubbed and the subtitled versions were available on the internet. The other reason relates to the length of the movie, which could provide grounds for the model under investigation to be tested. The movies needed to be relatively new (Late 1900s until now) and that it had to be popular and well-received by the public. The corpus for the analysis was comprised of English dialogues and its Persian dubs and subtitles.

\section{Procedure}

The movie was selected as already described in the 'material' section. The researcher began with a fully-attentive watching of the English, dubbed and subtitled versions of the movie. As mentioned before, the purpose of the study was to explore the differences and similarities between dubbing and subtitling in the English language movie. In order to do the research, the researcher followed a systematic procedure. After preparing the movie in DVD format, to ensure that the original dialogues match the translated ones, the researcher prepared the transcription of the movie and wrote every original frame (which was in English) and its translation in front of it. As this is a comparative study in the target language (Persian), the translation was studied in two forms. The first one is written (subtitle), and the second one is orally (dub), and both of them were compared to the original text (the main dialogues of the movie), sentence by sentence. The researcher closely compared source frame with its corresponding subtitles in target frame (A frame is a number of sentences or words that shown in a single scene. It was understood that it could be a word or a sentence). The lexical differences found and the researcher investigated why such differences existed at all. The researcher investigated the strategies of the translation like foreignization, and domestication based on Venuti's model, to understand under what condition such strategies were used. Another point, which was investigated during this research, was consideration of the both source and target language cultures. Some differences in translation forms (oral or written translation), is because of cultural differences in both countries (Iran and the United States). It is possible that some scenes, sentences, words, or other part of movie eliminated or changed. Then the whole process, from collecting data and grouping them, to their analysis was done manually. The unit of analysis was frame. The main source for the applied classification of this method was Newmark's classification (1991) of the culture-specific items [14] and Venuti's (2012) strategies of foreignization and domestication [15] and Van Dijk's (1999) idea about CDA in the part of Dominance [16].

\section{Design and Analysis}

As mentioned in the previous section, the data for this study were collected through comparing the dialogues of the original movie with subtitles of same movie and its dubbed dialogues. This helps to find the lexical differences and similarities between main, and dubbed and subtitled dialogues.

The researcher aimed to find if there was any incoherence between dubbing and subtitling. To achieve this, Newmark's classification (1991) of the culture-specific-items and Venuti's (2012) strategies of foreignization and domestication and Van Dijk's (1999) idea about CDA in the part of Dominance, were applied.

After preparing the scripts of the movie, every original frame was written in front of its translation. Then the whole dialogues of the original movie were compared with their subtitles and their dubbed dialogues. The examples of Venuti's strategies and Newmark's classification were written down and sorted. The percentages of occurrences of instances within a specific category or strategy were measured.

\section{Result}

Based on the examples given by Venuti (2012), mainly mentioned in the translator's invisibility, the researcher has adopted Newmark's classification (1991), of the culture specific items and Venuti's strategies of foreignization and domestication. After that, researcher used Van Dijk's (1999) idea about CDA (in part dominance) by using collected data. 
According to these strategies and classification, data collection and analysis carried out in a comparative descriptive framework. In other words, each original frame was transcribed and then compared with its translation to determine whether it is closer to the source-language or target-language culture. Finally, the researcher categorized these strategies, and calculated their frequencies.

RQ1: Results of analysis based on Venuti's strategies of domestication and foreignization
In this section, the strategies used by translators of the movie (both dubs and subtitles) were classified based on Venuti's strategies of domestication and foreignization. From 115 examples, derived from the movie, there are 70 examples of foreignization and 45 examples of domestication in subtitling of movie and there are 76 examples of domestication and 39 examples of foreignization in dubbing the movies. Table 1 shows the complete results of these classifications.

Table 1. Analysis of movie according to Venuti's strategies domestication and foreignization.

\begin{tabular}{|c|c|c|c|c|c|c|}
\hline \multirow{2}{*}{ Venuti's classification } & \multicolumn{2}{|c|}{ Frequency } & \multicolumn{2}{|c|}{ Total number } & \multicolumn{2}{|c|}{ Percentage } \\
\hline & Subtitles & Dubs & Subtitles & Dubs & Subtitles & Dubs \\
\hline Explanation & 11 & 5 & 115 & 115 & $9.50 \%$ & $4.34 \%$ \\
\hline Cultural equivalence & 20 & 44 & 115 & 115 & $17.34 \%$ & $38.26 \%$ \\
\hline Cultural adjustment & 1 & 5 & 115 & 115 & $0.80 \%$ & $4.34 \%$ \\
\hline \multicolumn{7}{|l|}{ Mestication } \\
\hline Addition & 5 & 2 & 115 & 115 & $4.34 \%$ & $1.73 \%$ \\
\hline Figurative expression for non-figurative ones & 1 & 0 & 115 & 115 & $0.80 \%$ & $0.00 \%$ \\
\hline Euphemism/Expurgation & 1 & 9 & 115 & 115 & $0.80 \%$ & $7.82 \%$ \\
\hline Syntactical adjustment & 4 & 10 & 115 & 115 & $3.47 \%$ & $8.69 \%$ \\
\hline \multicolumn{7}{|l|}{ Foreignization } \\
\hline Loan word/transliteration & 4 & 0 & 115 & 115 & $3.47 \%$ & $0.00 \%$ \\
\hline Calque translation & 0 & 0 & 115 & 115 & $0.00 \%$ & $0.00 \%$ \\
\hline Literal translation & 66 & 34 & 115 & 115 & $57.40 \%$ & $29.56 \%$ \\
\hline Total number & 115 & 115 & 115 & 115 & $100 \%$ & $100 \%$ \\
\hline
\end{tabular}

In the figure 1 we can see how much the dubbing and subtitling are different according to Venuti. Looking to this figure, we can understand easily that most differences are in the cultural equivalence and literal translation.

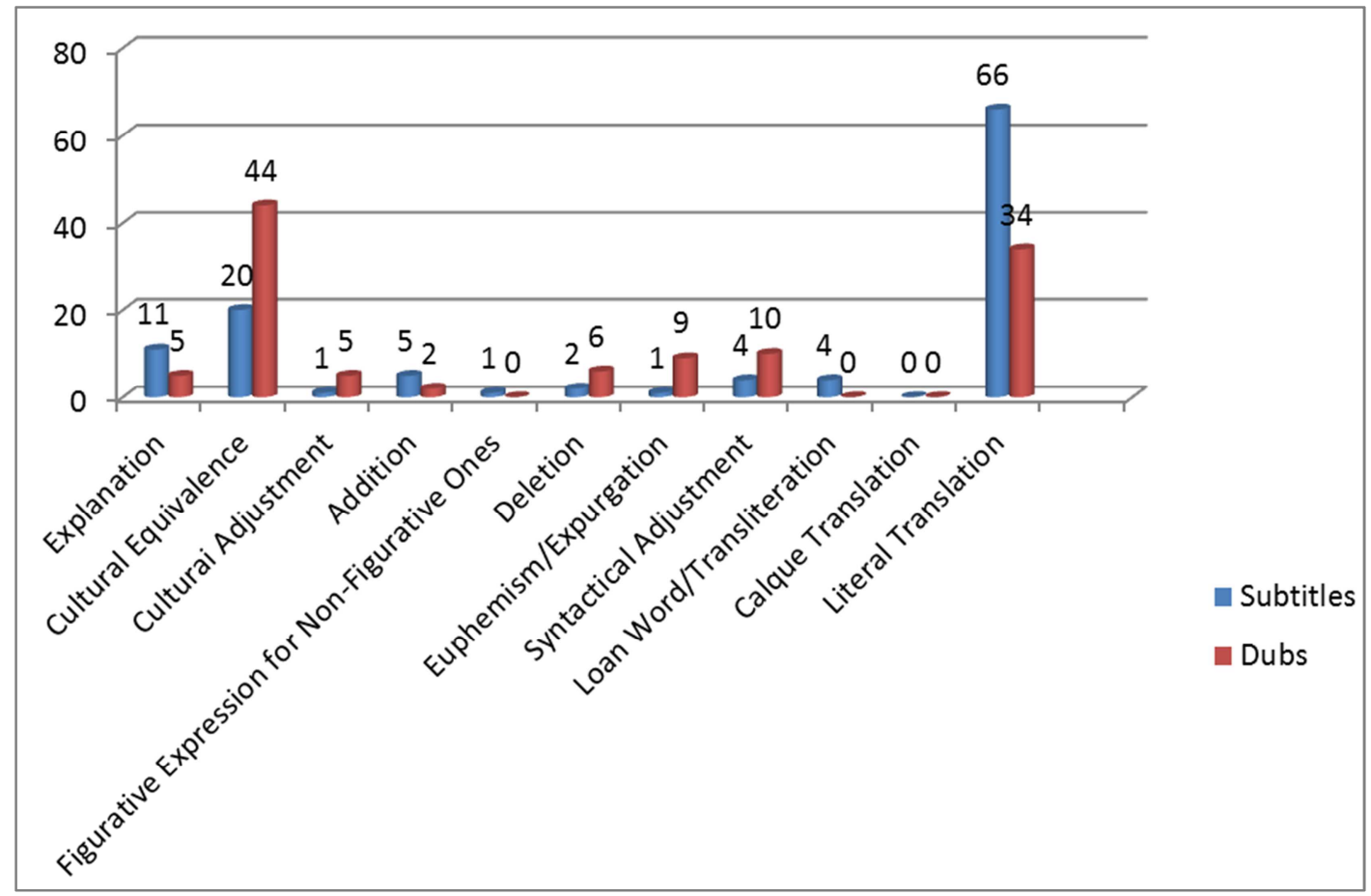

Figure 1. Analysis of movie according to Venuti's strategies domestication and foreignization.

In figure 1 comparison of the differences and similarities between foreignization and domestication during dubbing and subtitling of the movie can be seen obviously. The difference between them is more in dubbing and less in subtitling. So, it can be concluded that the translator paid attention to the literal translation and cultural equivalence simultaneously in subtitling the movie in order to have more exact translation; while in the dubbing, paying attention to cultural equivalence was more important. Because translator wanted to make the movie visible by every group of peoples in Iran. 


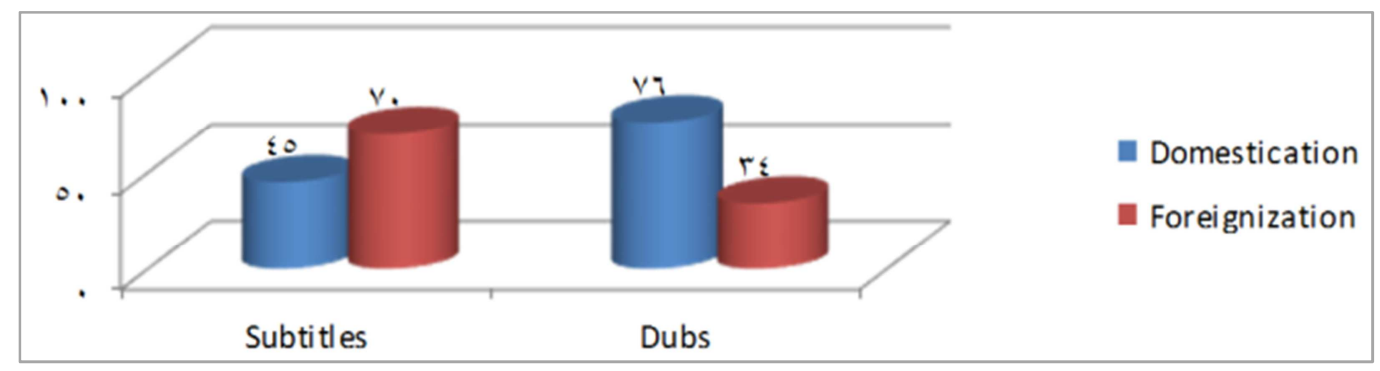

Figure 2.Analysis of the movie according to Venuti's strategies.

RQ2: Results of Analysis based on Newmark's Classification of Culture-specific items

The movie was analyzed again, but this time according to the Newmark's classification of culture-specific items and the results shown in the following Table.

Table 2.Analysis of movie according to Newmark's classification of culture-specific items.

\begin{tabular}{|c|c|c|c|c|c|c|}
\hline \multirow{2}{*}{ Newmark's classification } & \multicolumn{2}{|c|}{ Frequency } & \multicolumn{2}{|c|}{ Total number } & \multicolumn{2}{|c|}{ Percentage } \\
\hline & Subtitles & Dubs & Subtitles & Dubs & Subtitles & Dubs \\
\hline Ecology & 7 & 7 & 115 & 115 & $6.08 \%$ & $6.08 \%$ \\
\hline Material cultures & 15 & 13 & 115 & 115 & $13.04 \%$ & $11.30 \%$ \\
\hline Social cultures & 14 & 13 & 115 & 115 & $13.77 \%$ & $11.30 \%$ \\
\hline Organizations & 40 & 39 & 115 & 115 & $34.78 \%$ & $33.91 \%$ \\
\hline Gestures and habits & 0 & 0 & 115 & 115 & $0.00 \%$ & $0.00 \%$ \\
\hline Euphemism/Expurgation & 0 & 5 & 115 & 115 & $0.00 \%$ & $4.34 \%$ \\
\hline Syntactic features & 38 & 37 & 115 & 115 & $33.04 \%$ & $32.17 \%$ \\
\hline Total & 115 & 115 & 115 & 115 & $100 \%$ & $100 \%$ \\
\hline
\end{tabular}

In the following figure, the differences and similarities between Newmark's classifications of culture-specific items are shown.

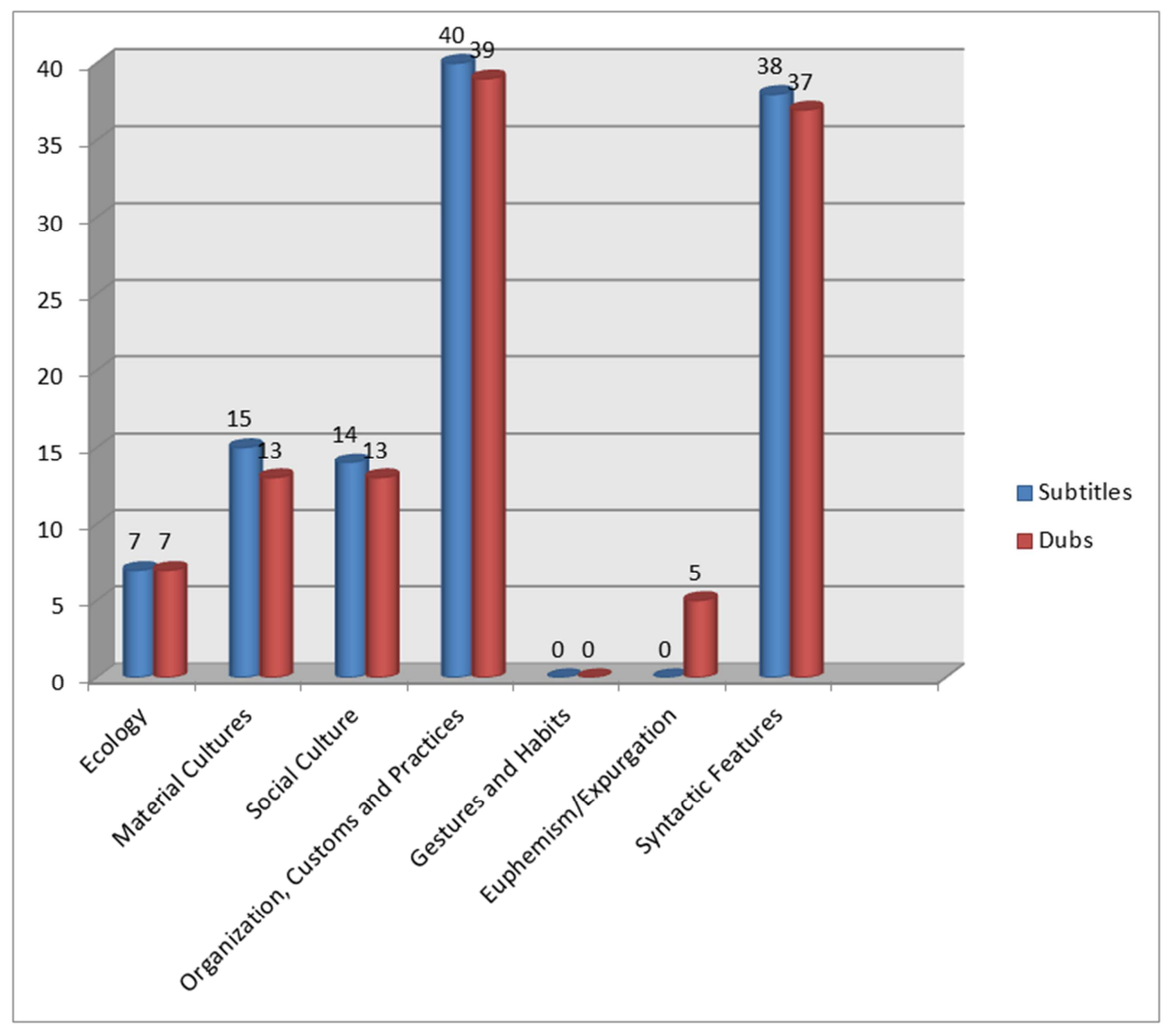

Figure 3. Analysis of the movie according to Newmark's classification of culture-specific items. 
The most obvious differences are for literal translation in which, in subtitling, it has $57.44 \%$ and in dubbing it has $29.56 \%$ of whole percent. After that, cultural equivalence is located, in subtitling $17.34 \%$ and in dubbing $38.26 \%$. Explanation is in the third step, in subtitling 9.50\% and in dubbing $4.34 \%$. Next one is euphemism/expurgation that in subtitling is $0.80 \%$ and in dubbing is $7.82 \%$. Finally, syntactical adjustment, that in subtitling is $3.47 \%$ and in dubbing is $8.69 \%$, is located.

However, the most obvious similarities, in both dubbing and subtitling, are for calque translation that is nothing. After that, figurative expression for non-figurative one is located, in subtitling $0.80 \%$ and in dubbing $0.00 \%$. Addition is in third step, in subtitling $4.34 \%$ and in dubbing $1.73 \%$. Next one is cultural adjustment, in subtitling $0.80 \%$ and in dubbing $4.34 \%$. Next, we have loan word/transliteration, in subtitling $3.47 \%$ and in dubbing $0.00 \%$. Finally, deletion, that in subtitling is $1.73 \%$ and in dubbing is $5.21 \%$, is located.

RQ3: Results of analysis based on Van Dijk's idea about $C D A$ (in the part of dominance)

According to data, collected from the movie, and dominance (a part of CDA) defined by Van Dijk, it can be concluded that the dominant strategy used in subtitling is to be faithful to the source language (literal translation) and foreignization. Translator tries to import foreign culture to target language country. Sometimes, when it is necessary, translator uses cultural equivalences, just because of some dialogues be meaningful in the target language, but not to change their meaning completely.

In the contrary, the dominant strategy used in dubbing is domestication. Translator tries to change or even eliminate some swearwords to make some special dialogues suitable in the target language culture.

\section{Discussion and Limitation}

The present study investigated the lexical differences and similarities in translating of the English language movies into Persian. The adopted models and comparing mentioned percentages can conclude that according to the strategies of translation, classified by Venuti, there are some similarities and differences between dubbing and subtitling of the movie. According to Venuti strategies, in subtitling, Iranian translators use foreignization more than domestication. While; in dubbing, they use domestication more than foreignization.

Again, by comparing mentioned percentages, according to New mark's classification, dubbing and subtitling are almost as same and cannot find a considerable different between them. The only negligible different, in dubbing, is for euphemism/expurgation, in which translator decided to eliminate some lexical items because of restrictions existed in the target language country (Iran) of course (some parts of the movie are censored completely because of sex matters and are not translated at all, so can count them in the euphemism/ expurgation part). As a result, similarities are more in this part.

It is interesting that in both, dubbing and subtitling, the item of gestures and habits are nothing $(0.00 \%)$. Moreover, in both of them Organization, Customs and Practices is first and after that syntactic feature is located in the second step.

Up to here, the researcher just compared dubbing and subtitling according to Newmark's classification of cultural specific items and Venuti's strategies of domestications and foreignization, but the main aim of current study is comparing dubbing and subtitling through CDA, introduced by Van Dijk, in the part of dominance.

As Nida (2000)states, dominance is defined as the exercise of social power by elites, institutions or groups, that results in social inequality, including political, cultural, class, ethnic, racial and gender inequality. This reproduction process may involve such different modes of discourse power relations as the more or less direct or overt support, enactment, representation, legitimation, denial, mitigation or concealment of dominance, among. More specifically, critical discourse analysis wants to know what structures, strategies or other properties of text, talk, verbal interaction or communicative events play a role in these modes of reproduction [17].

Watching movies in their source language is not ordinary by all peoples. So subtitling is not necessary for all people in target language. In the contrary, in target country, there are a lot of people who love to watch movie in its source language. Persons who love to watch these kinds of movies, should have some characteristics, such as: to be old enough to read the subtitle in its normal speed or; having interest and respective knowledge of source language or; having interest of watching foreign language movies, and... Therefore, in these positions, translating of the movie is easier task and translator is freer to be faithful.

Moreover, translator can be sure enough that children will not encounter with impolite dialogues in the target language. If they, accidentally, hear this kind of dialogues, they cannot understand their mean completely. So, translating swearwords or even sexy words, is not so hard to translators.

Frames will not eliminate in the main movie when subtitling. Therefore, there is no censorship in the movie and translator has to translate all dialogues, without any expurgation.

On the contrary, in dubbing, because of some restrictions mentioned above, the translator is not free enough to translate everything completely.

\section{Conclusion}

Comparison of the original English movie with its Persian subtitles and dubs gave rise to interesting results. The results show that within the realm of Newmark classification, cultural specific items have been used in subtitles and dubs of the movie, but not equally. As indicated, according to Newmark classification, the total amount of Organization, Customs and Practices (in both dubbing and subtitling) enjoys the highest 
frequency (34.78\% in subtitling and 33.91 in dubbing) and percentage and amount of gestures and habits gets the least frequency $(0.00 \%$ for both dubbing and subtitling).

As shown before, according to Venuti's strategies, in the subtitles of the movie, the total amount of literal translation $(57.4 \%)$ has the most frequency, and in the dubs of the movie, the total amount of the cultural equivalence (38.26\%) has the most frequency. In addition, in both dubbing and subtitling, the calque translation $(0.00 \%)$ has the least frequency. It is clear and worth to noting, the amount of foreignization $(61.22 \%)$ in the movie's subtitles is higher, compared to domestication (39.78\%). In addition, the amount of the domestication (70.44\%) in the movie's dubs is higher, compared to foreignization $(29.56 \%)$.

Comparing these results can say, according to CDA, the dominant strategy used in subtitling is foreignization and dominant strategy used in dubbing is domestication.

This research has some implications. Audiovisual translation (AVT) is a new field of study and much research can be done in this field. The new technologies and movements of the world also affect Iran in the field of languages. Therefore, these movements intensify the need to accept and emphasize that audiovisual translation can be studied as a course in translation studies or even other fields at universities. This research can also be used in translation training program. Translators can use the results of the study to do research about audiovisual translation in other genres, fields and even enjoy the results of this study. In addition, the novice translators can use these strategies and sub-strategies to improve their abilities in translating and learning foreign languages.

Translators must be aware of the fact that the heart of their tasks is not to translate texts, but to translate cultures. They should know that misinterpretations occur when they do not consider culture. If they translate the cultural words literally, since the meaning would be distorted, the target language readers or audiences will be culturally shocked.

In the process of this study, the researcher could not easily differentiate between mistranslations and the strategies of domestication and foreignization. In other words, it was very difficult to judge whether the items were deliberately chosen or because of the lack of knowledge. One reason can be that translators translate films in a rush and only through listening to the sound tracks. Besides, each language has unique characteristics and one of the main translation problems is to find possible strategies, and analyze specific translations. Hence, it would be helpful for translators to find the norms that govern the choice of translation strategy. In other words, they would be more successful if they know which strategies are used more and are accepted by competent translators. Moreover, from Venuti's point of view, it seems so necessary to make translation students aware of the importance of preserving cultural and linguistic diversities of any language. What is more important is the awareness of the translators in selecting a text, the genre, its author and in adopting a translation method.

The findings of the present work have the potentialities of acting as a tool to understanding the phenomena of dubbing and subtitling issues better. From 1990s onward, an increasing interest has been paid to the process- oriented research programs. Accordingly, translation strategies play a significant role in this regard. Thus, it is hoped that the present thesis project would be able to informing work provided on the issue[15].

\section{References}

[1] Venuti, L. (2017). The translator's invisibility: A history of translation: Routledge.

[2] Dries, J. (1995). Dubbing and subtitling: Guidelines for production and distribution: Europ. Medieninst.

[3] Gottlieb, H. (2004). Language-political implications of subtitling. BENJAMINS TRANSLATION LIBRARY, 56, 83100 .

[4] Ghaemi, F., \& Benyamin, J. (2011). Strategies used in the translation of interlingual subtitling. Journal of English Studies, 1, 39-49.

[5] Gottlieb, H. (1992). Subtitling-a new university discipline. Paper presented at the Teaching Translation and Interpreting: Training, Talent and Experience. Papers from the First Language International Conference, Elsinore, Denmark 31 May-2 June 1991.

[6] Dastjerdi, H. V., \& Rahekhoda, R. (2010). Expansion in Subtitling: The Case of Three English Films with Persian Subtitles. Journal of Universal Language, 11(1), 7-27.

[7] De Linde, Z., \& Kay, N. (2016). The semiotics of subtitling: Routledge.

[8] Pelsmaekers, K., \& Van Eesien, F. (2002). Subtitling irony: Blackadder in Dutch. The Translator, 8(2), 241-266.

[9] Sharei, S., Yazdanmehr, E., \& Esfahani, A. F. P. (2017). Translation of Anthroponyms in Children's Cartoons: A Comparative Analysis of English Dialogues and Persian Subtitles. Language and Translation, 7(4), 21-31.

[10] Gambier, Y. (1994). Multimédia et médiation: quel défis. Problemi e tendenze delladidatticadell'interpetazione e dellatraduzione, 67-79.

[11] Fernandes, L. (2006). Translation of names in children's fantasy literature: Bringing the young reader into play. New voices in translation studies, 2(2), 44-57.

[12] Bertills, Y. (2003). Beyond identification: proper names in children's literature.

[13] Pérez-González, L. (2007). Appraising dubbed conversation: Systemic functional insights into the construal of naturalness in translated film dialogue. The Translator, 13(1), 1-38.

[14] Newmark, P. (1991). About translation (Vol. 74): Multilingual matters.

[15] Venuti, L. (2012). The translation studies reader: Routledge.

[16] Van Dijk, T. A. (1999). Critical discourse analysis and conversation analysis: Sage Publications.

[17] Nida, E. (2000). Principles of Correspondence. 1964. Bassnet, Susan. Translation Studies. 


\section{Biography}

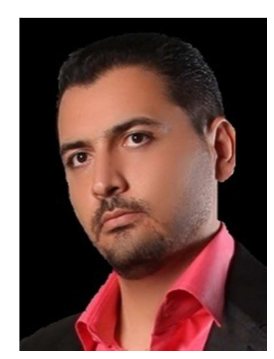

Noie Saber Received his M.A. in English Translation from Islamic Azad UniversitySouth Branch Tehran, Iran. He is interested in teaching language methodology specially TBLT, and EFL materials preparation. He is a written translator.

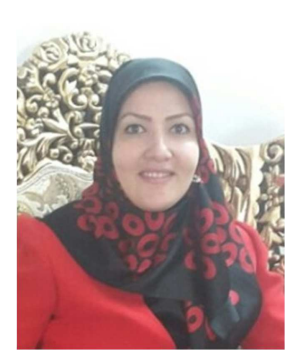

Jafar Pour Fariba graduated with a master degree in the field of Persian Literature from the Islamic Azad University-North Branch Tehran, Iran. She is interested in poetry and is prime manager in Tehran municipality. She is a tentative editor of Persian books. 